УДК 519.624.2

\title{
АСИМПТОТИЧЕСКОЕ РЕШЕНИЕ ЗАДАЧИ КОНВЕКТИВНОЙ ДИФФУЗИИ ОКОЛО КАПЛИ В ПОТОКЕ С УЧЕТОМ ОБЪЕМНОЙ ХИМИЧЕСКОЙ РЕАКЦИИ
}

\author{
Кириллова Ирина Владимировна \\ студентка института физики, \\ математики, цифровых и нанотехнологий \\ ФГБОУ ВО БГПУ им. М.Акмуллы \\ Научный руководитель: Ахметов Рустям Гилимович \\ д. ф-м. н., профессор кафедры ПиВМ \\ ФГБОУ ВО БГПУ им. М.Акмуллы
}

Аннотация: Статья посвящена краевой задаче квазилинейного эллиптического уравнения в частных производных. Уравнение описывает процесс конвективной диффузии около капли в потоке с учетом объемной химической реакции. Для рассматриваемой задачи асимптотическое разложение решения получается около сферической капли.

Ключевые слова: краевая задача, метод согласования, седловая точка, диффузионный пограничный слой, асимптотическое разложение.

\section{AN ASYMPTOTIC SOLUTION TO THE PROBLEM OF CONVECTIVE DIFFUSION NEAR A DROP IN THE FLOW, TAKING INTO ACCOUNT THE VOLUME CHEMICAL REACTION}

Kirillova Irina Vladimirovna

Scientific adviser: Akhmetov Rustyam Gilimovich

\begin{abstract}
The article is devoted to the boundary value problem of a quasilinear elliptic partial differential equation. The equation describes the process of convective diffusion near a drop in the flow, taking into account the volume chemical reaction. For the problem under consideration, the asymptotic expansion of the solution is obtained near a spherical drop.

Key words: boundary value problem, matching method, saddle point, the diffusion boundary layer, asymptotic expansion.
\end{abstract}




\section{1. Постановка задачи}

Рассматривается краевая задача

$$
\begin{gathered}
\Delta u=P e(\bar{V}, \nabla) u+k_{v} F(u), \\
u=1 \text { при } r=1 ; u \rightarrow 0 \text { при } r \rightarrow \infty,
\end{gathered}
$$

где $\bar{V}=\left(V_{r}, V_{\theta}, 0\right), V_{r}=\frac{1}{r^{2} \sin \theta} \frac{\partial \psi}{\partial \theta}, V_{\theta}=-\frac{1}{r \sin \theta} \frac{\partial \psi}{\partial r}, \psi(r, \theta)-$ функция тока, $r, \theta-$ сферические координаты $(0 \leq \theta \leq \pi), \Delta$ - оператор Лапласа, $P e$ - число Пекле и $k_{v}$ - константа скорости объемной химической реакции. Угол $\theta$ отсчитывается от направления потока на бесконечности.

В линейном случае, то есть $F(u) \equiv u$, задача рассматривается в работе [1]. Функция тока имеет вид:

$$
\psi(r, \theta)=\frac{\sin ^{2} \theta(r-1)\left(2 r-\frac{\lambda}{\lambda+1}\left(1+\frac{1}{r}\right)\right)}{4}, \text { где } \lambda \text { - const. }
$$

Предположим, что

$$
F(u) \in C^{k}, F(0)=0, F^{\prime}(0)=0, F^{\prime \prime}(0)>0, k>9,
$$

и имеет разложение

$$
F(u)=u^{2}+F_{2} u^{4}+F_{3} u^{6}+F_{4} u^{8}+O\left(u^{10}\right) \text { для } u \rightarrow 0 .
$$

\section{2. Диффузионный пограничный слой}

В диффузионном слое введем параметр $\varepsilon=P e^{-\frac{1}{2}}$ и перепишем уравнение (1.1) в виде:

$$
L_{\varepsilon} u-\mu F(u)=\varepsilon^{2} \Delta u-\frac{1}{r^{2} \sin \theta}\left(\frac{\partial u}{\partial r} \frac{\partial \psi}{\partial \theta}-\frac{\partial u}{\partial \theta} \frac{\partial \psi}{\partial r}\right)-\mu F(u)=0 .
$$

В том случае, когда введенный параметр будет равен нулю, уравнение (2.1) имеет седловые точки: $O_{1}(1, \pi), O_{2}(1,0)$.

Вблизи поверхности капли естественные переменные $x=\varepsilon^{-1}(\lambda+1)^{-\frac{1}{2}}(r-$ 1), $\theta$. Асимптотика решения строится как

$$
u(x, \theta, \varepsilon)=u_{0}(x, \theta)+O(\varepsilon) .
$$

Для определения $u_{0}(x, \theta)$ в переменных $x, \theta$ с использованием (1.1) - (1.2), (2.1) получаем задачу:

$$
\begin{gathered}
\frac{\partial^{2} u_{0}}{\partial x^{2}}-x \cos \theta \frac{\partial u_{0}}{\partial x}+\sin \theta \frac{\partial u_{0}}{\partial \theta}-\mu(\lambda+1) F\left(u_{0}\right)=0, \\
u_{0}(0, \theta)=1 ; u_{0}(x, \theta) \rightarrow 0 \text { при } x \rightarrow \infty .
\end{gathered}
$$

Асимптотика решения $u_{0}(x, \theta)$ при $\theta \rightarrow \pi$ изучена в работе [2]. 


\section{3. Асимптотика функции $\boldsymbol{u}_{\mathbf{0}}(\boldsymbol{x}, \boldsymbol{\theta})$ при $\boldsymbol{\theta} \rightarrow \mathbf{0}$}

Асимптотическое разложение $u_{0}(x, \theta)$ при $\theta \rightarrow 0$ будем искать в виде:

$$
u_{0}(x, \theta)=v_{0}(x)+O\left(\theta^{2}\right),
$$

где функция $v_{0}(x)$ построена как решение задачи

$$
\begin{gathered}
v_{0}^{\prime \prime}(x)-x v_{0}^{\prime}(x)-\mu(\lambda+1) F\left(v_{0}(x)\right)=0, \\
v_{0}(0)=1 ; v_{0}(x)=O(1) \text { при } x \geq 0 .
\end{gathered}
$$

Теорема. Пусть для функиии $F(u)$ справедливы условия (1.4), (1.5),тогда для решения задачи (3.2)-(3.3) существует асимптотика при $x \rightarrow \infty$, имеющая вид:

$$
v_{0}(x)=\sum_{k=1}^{n} \frac{C_{o, k}}{(\mu \ln (x)+c)^{k}}+\frac{1}{x^{2}} \sum_{k=2}^{m} \frac{C_{1, k}}{(\mu \ln (x)+c)^{k}}+O\left(\frac{1}{x^{4}}\right)
$$

для некоторого $c \in R$, где

$$
\begin{aligned}
& \mathrm{C}_{0,1}=1 ; \mathrm{C}_{0,2}=\text { const } ; \mathrm{C}_{0,3}=\mathrm{C}_{0,2}^{2}+F_{2} ; \mathrm{C}_{0,4}=\frac{1}{2}\left(2 \mathrm{C}_{0,2} C_{0,3}+4 F_{2} C_{0,2}\right) ; \\
& \mathrm{C}_{0,5}=\frac{1}{3}\left(2 \mathrm{C}_{0,2} C_{0,4}+6 F_{2} C_{0,2}^{2}+4 F_{2} C_{0,3}+F_{3}+C_{0,3}^{2}\right) ; \\
& \mathrm{C}_{0,6}=\frac{1}{4}\left(2 \mathrm{C}_{0,2} C_{0,5}+2 C_{0,3} C_{0,4}+4 F_{2} C_{0,2}^{3}+12 F_{2} C_{0,2} C_{0,3}+4 F_{2} C_{0,4}+6 F_{3} C_{0,2}\right) ; \\
& \mathrm{C}_{0,7}=\frac{1}{5}\left(2 \mathrm{C}_{0,2} C_{0,6}+2 C_{0,3} C_{0,5}+F_{2} C_{0,2}^{4}+6 F_{2} C_{0,3}^{2}+12 F_{2} C_{0,2}^{2} C_{0,3}+\right. \\
& \left.12 F_{2} C_{0,2} C_{0,4}+4 F_{2} C_{0,5}+15 F_{3} C_{0,2}^{2}+6 F_{3} C_{0,3}+F_{4}+C_{0,4}^{2}\right) ; \\
& C_{1,2}=-\frac{\mu}{2} ; \quad C_{1,3}=-\frac{\mu}{2}\left(2 C_{0,2}+2 \mu\right) ; \quad C_{1,4}=-\frac{\mu}{2}\left(3 C_{0,3}+6 \mu C_{0,2}+C_{1,3}-\right. \\
& \left.2 C_{1,2} C_{0,2}\right) ; \\
& C_{1,5}=-\frac{\mu}{2}\left(4 C_{0,4}+12 \mu C_{0,3}+2 C_{1,4}-2 C_{1,3} C_{0,2}-2 C_{1,2} C_{0,3}-4 F_{2} C_{1,2}\right) ; \\
& C_{1,6}=-\frac{\mu}{2}\left(5 C_{0,5}+20 \mu C_{0,4}+3 C_{1,5}-2 C_{0,4} C_{1,2}-2 C_{0,2} C_{1,4}-2 C_{0,3} C_{1,3}-\right. \\
& \left.12 F_{2} C_{0,2} C_{1,2}-4 F_{2} C_{1,3}\right) . \\
& v_{0}(x)>0 ; v_{0}^{\prime}(x)<0 \text { для } x>0 .
\end{aligned}
$$

Доказательство проводится аналогично доказательству теоремы 1 работы [Error! Reference source not found.]. Решение $v_{0}(x)$ ищем в виде:

$$
v_{0}(x)=v_{n}(x)+w(x)
$$


где $v_{n}(x)=\sum_{i=0}^{m} \sum_{k=1}^{n} C_{i, k} x^{-2 i}\left(\frac{1}{\mu \ln (x)+C}\right)^{k}, \quad$ при $x \geq 2, n \geq 8, m \geq 1, v_{n}(x) \in$ $C^{2}(x \geq 0)$. Подставляя сумму (3.6) в уравнение (3.2), получаем задачу $L w-\mu\left(F\left(w+v_{3}\right)-F\left(v_{3}\right)\right)=H_{3}(x)$,

$w(x) \rightarrow 0, w^{\prime}(x) \rightarrow 0, \quad$ для $\quad x \rightarrow \infty, \quad$ где $\quad\left|H_{3}(x)\right| \leq M(\ln (x))^{-9}+$ $M x^{-2}(\ln (x))^{-4}+M x^{-4}$.

Аналогично теореме 1 [3] для решения уравнения (3.7) получаем неравенство:

$$
|w(x)| \leq M_{1}(\ln (x))^{-9}+M_{1} x^{-2}(\ln (x))^{-4}+M_{1} x^{-4}
$$

для некоторого $M_{1}>0$.

\section{4. Область задней критической точки}

Решение в окрестности задней критической точки строится в переменных $x=$ $\varepsilon^{-1}(\lambda+1)^{-\frac{1}{2}}(r-1), \xi=\varepsilon^{-1}(\lambda+1)^{-\frac{1}{2}} \theta$. Главный член строится как решение уравнения

$$
\begin{aligned}
\frac{\partial^{2} u^{(3)}(x, \xi)}{\partial \xi^{2}} & +\frac{\partial^{2} u^{(3)}(x, \xi)}{\partial x^{2}}-t \frac{\partial u^{(3)}(x, \xi)}{\partial x}+\left(\frac{\xi}{2}+\frac{1}{\xi}\right) \frac{\partial u^{(3)}(x, \xi)}{\partial \xi} \\
& -\mu(\lambda+1) F\left(u^{(3)}(x, \xi)\right)=0
\end{aligned}
$$

удовлетворяющее граничным условиям

$$
u^{(3)}(0, \xi)=1 ; \frac{\partial u^{(3)}(x, \xi)}{\partial \xi}=0 \text { при } \xi=0
$$

и условию согласования:

$$
u^{(3)}(x, \xi)-v_{0}(x) \rightarrow 0 \text { при } \xi \rightarrow \infty .
$$

Функция $v_{0}(x)$, построенная в теореме 1 , удовлетворяет всем требуемым условиям (4.1) - (4.3).

\section{5. Численное решение}

Заметим, что постоянная $c$ - произвольная. Уравнение (1.1) перепишем в виде системы:

$$
\left\{\begin{array}{c}
y^{\prime}(\mathrm{t})=\mathrm{z}(\mathrm{x}) \\
\mathrm{z}^{\prime}(\mathrm{x})=\mathrm{xz}(\mathrm{x})+\mu \mathrm{F}(\mathrm{u})
\end{array}\right.
$$

Из условий устойчивости явной схемы Рунге-Кутты следует, что начальные условия необходимо задавать при большом значении х и интегрировать назад (то есть, с шагом $h<0$ ), например, в точке $\mathrm{x}_{0}=150$ :

$$
\mathrm{y}(150)=\mathrm{y}_{0}, \mathrm{z}(150)=\mathrm{z}_{0},
$$


где

$$
\begin{aligned}
& \mathrm{y}_{0} \approx \frac{\mathrm{C}_{0,1}}{(\mu * \ln (x)+c)}+\frac{\mathrm{C}_{0,2}}{(\mu * \ln (x)+c)^{2}}+\frac{\mathrm{C}_{0,3}}{(\mu * \ln (x)+c)^{3}} \\
& +\frac{\mathrm{C}_{0,4}}{(\mu * \ln (x)+c)^{4}}+\frac{\mathrm{C}_{0,5}}{(\mu * \ln (x)+c)^{5}}+\frac{\mathrm{C}_{0,6}}{(\mu * \ln (x)+c)^{6}} \\
& +\frac{\mathrm{C}_{0,7}}{(\mu * \ln (x)+c)^{7}}+\frac{\mathrm{C}_{0,8}}{(\mu * \ln (x)+c)^{8}}+\cdots \\
& +\frac{1}{\mathrm{x}^{2}}\left(\frac{\mathrm{C}_{1,2}}{(\mu * \ln (x)+c)^{2}}+\frac{\mathrm{C}_{1,3}}{(\mu * \ln (x)+c)^{3}}+\frac{\mathrm{C}_{1,4}}{(\mu * \ln (x)+c)^{4}}\right. \\
& +\frac{\mathrm{C}_{1,5}}{(\mu * \ln (x)+c)^{5}}+\frac{\mathrm{C}_{1,6}}{(\mu * \ln (x)+c)^{6}}+\frac{\mathrm{C}_{1,7}}{(\mu * \ln (x)+c)^{7}} \\
& +\cdots) \\
& \mathrm{z}_{0} \approx-\frac{\mu * \mathrm{C}_{0,1}}{x(\mu * \ln (x)+c)^{2}}-\frac{2 * \mu * \mathrm{C}_{0,2}}{x(\mu * \ln (x)+c)^{3}}-\frac{3 * \mu * \mathrm{C}_{0,3}}{x(\mu * \ln (x)+c)^{4}} \\
& -\frac{4 * \mu * \mathrm{C}_{0,4}}{x(\mu * \ln (x)+c)^{5}}-\frac{5 * \mu * \mathrm{C}_{0,5}}{x(\mu * \ln (x)+c)^{6}}-\cdots \\
& -\frac{2}{x^{3}}\left(\frac{C_{1,2}}{(\mu * \ln (x)+c)^{2}}+\frac{C_{1,3}}{(\mu * \ln (x)+c)^{3}}+\cdots\right) \\
& -\frac{\mu}{x^{3}}\left(\frac{2 * C_{1,2}}{(\mu * \ln (x)+c)^{3}}+\frac{3 * C_{1,3}}{(\mu * \ln (x)+c)^{4}}+\cdots\right)
\end{aligned}
$$

Постоянные a, b задаются в качестве интервала для нахождения константы $c$ методом половинного деления. Выберем $a=0.0001, b=10$. После задания вида функции $\mathrm{F}(\mathrm{u})$ задачу (1.1) - (1.2) решаем численно.

Рассмотрим случай, когда $\mathrm{F}(\mathrm{u})=\mathrm{u} * \operatorname{Sin}(\mathrm{u})$, находя последовательные приближения $\mathrm{c}_{0, \mathrm{n}}, \mathrm{n}=2,3, \ldots$ и $\mathrm{c}_{1, \mathrm{n}}, \mathrm{n}=2,3, \ldots$ для $\mathrm{c}$ при помощи использования метода половинного деления.

Для численного решения задачи (5.1) - (5.2) был использован метод Рунге-Кутта.

Результаты вычислений для различных $\mu$ :

$$
\begin{gathered}
\mu=0.5: \mathrm{c}=6.1536 ; \mathrm{y}=0.99997 ; \mathrm{z}=-0.00517 . \\
\mu=1: \mathrm{c}=3.64813 ; \mathrm{y}=1.0001 ; \mathrm{z}=-0.01034 . \\
\mu=1.5: \mathrm{c}=1.14267 ; \mathrm{y}=1.0002 ; \mathrm{z}=-0.01552 .
\end{gathered}
$$


С помощью компьютерного моделирования в данном случае мы решили задачу о нахождении решения дифференциального уравнения $u^{\prime \prime}-x u^{\prime}-$ $\mu F(u)=0, \quad$ которое удовлетворяет краевым условиям $u(0)=1, u(x)=$ $O(1)$ на интервале $(0.0001 ; 10)$ при $x \geq 0$ для различных значений $\mu$.

\section{Список литературы}

1. Zhivotyagin A.F., Influence of a Homogeneous Chemical Reaction on the Distribution of Concentration in a Diffusion Wake of a Droplet // Vestn. Mosk. Gos. Univ. Ser. 1. Mat. Mekh. 1980. №6. P. 73-78.

2. R. G. Akhmetov, Asymptotics of solution for a problem convective diffusion with volume reaction near a spherical drop, in: Proceedings of the Steklov institute of mathematics, vol. Suppl. 2003, pp. S8-S12.

3. R. G. Akhmetov, The asymptotic expansions of the solution for the boundary value problem to a convective diffusion equation with volume chemical reaction near a spherical drop: Commun Nonlinear Sci Numer Simulat, 16 (2011), 2308-2312. 